\title{
Proposição de uma teoria substantiva para o processo de acreditação hospitalar: o modelo "comprometimento com o cuidado"
}

\author{
ANDREIA MARIA BERTO ${ }^{1}$ \\ ROLF HERMANN ERDMANN ${ }^{2}$ \\ VIVIAN OSMARI UHLMANN ${ }^{3}$
}

\begin{abstract}
Resumo
Este estudo apresenta uma teoria substantiva que se propõe a explicar como interagem e que fatores influenciam os elementos envolvidos no processo de implantação, manutenção e evolução da acreditação hospitalar. A pesquisa foi realizada entre os anos de 2015 e 2017 , por meio do case do Hospital Regional Público da Transamazônica (HRPT), localizado em Altamira (PA), região Norte do Brasil. Entrevistas, análise documental e observação participante ocorreram de forma longitudinal num período de 24 meses, espaço de tempo que compreendeu a manutenção do nível 2 da acreditação hospitalar no HRPT, bem como a evolução ao nível 3 e sua manutenção. Sob um viés construtivista e qualitativo, o levantamento de dados foi dividido em 5 rodadas, conforme as diretrizes da grounded theory na vertente de Charmaz, e envolveu 56 participantes. O modelo proposto, denominado "comprometimento com o cuidado", se mostrou uma alternativa integradora e sistêmica à compreensão de como ocorre a acreditação hospitalar na organização estudada. Sua estrutura é constituída por 3 categorias centrais "a forma como trabalhamos", "acreditação como instrumento de ação" e "amor à causa" -, 1 categoria de suporte - "desenvolvimento dos profissionais de saúde" - e 2 categorias de resultado - "excelência nos resultados" e "cuidado centrado no paciente". Esses achados dialogaram com o pensamento complexo e o estudo das instituições.
\end{abstract}

Palavras-chave: Acreditação hospitalar. Teoria substantiva. Hospital público.

\section{Proposition of a substantive theory for the hospital accreditation process: the "commitment to care" model}

\begin{abstract}
This study presents a substantive theory that aims to explain the factors that interact and influence the elements involved in the implantation, maintenance, and evolution of hospital accreditation. The study was carried out between 2015 and 2017, through the case of the Transamazonica Regional Public Hospital (HRPT), located in Altamira (state of Pará), in the northern region of Brazil. Interviews, documentary analysis, and participant observation were conducted longitudinally over a period of 24 months, a period that comprised the maintenance of level 2 of hospital accreditation in HRPT, and the evolution at level 3 and its maintenance at this level. Under a constructivist and qualitative bias, data collection was divided into five rounds, according to the guidelines of Charmaz grounded theory, and involved 56 participants. The proposed model, called "Commitment to Care," was an integrative and systemic alternative to understanding how hospital accreditation occurs in the organization studied. Its structure consists of three central categories: "The way we work," "Accreditation as an instrument of action," and 'Love to the cause'; a category of support: 'Development of health professionals'; and two outcome categories: "Excellence in Outcomes" and "Patient-Centered Care." These findings were in dialogue with complex thinking and the study of institutions.
\end{abstract}

Keywords: Hospital Accreditation. Substantive theory. Public hospital.

\section{Propuesta de una teoría sustantiva para el proceso de acreditación de hospitales: el modelo "compromiso con} el cuidado

\section{Resumen}

Este estudio presenta una teoría sustantiva que tiene como objetivo explicar los factores que interactúan e influyen en los elementos involucrados en el proceso de implantación, mantenimiento y evolución de la acreditación hospitalaria. La investigación se llevó a cabo entre 2015 y 2017, a través del caso del Hospital Regional Público da Transamazônica (HRPT), ubicado en Altamira (estado do Pará), norte de Brasil. Las entrevistas, el análisis de documentos y la observación participante se llevaron a cabo longitudinalmente durante un período de 24 meses. Este período de tiempo abarcó el mantenimiento del nivel 2 de la acreditación hospitalaria en el HRPT, así como la evolución al nivel 3 y su mantenimiento en ese nivel. Bajo un sesgo constructivista y cualitativo, el levantamiento de datos se dividió en cinco rondas, según las directrices de la teoría fundamentada de Charmaz, e involucró a 56 participantes. El modelo propuesto, Ilamado Compromiso con el Cuidado, demostró ser una alternativa integradora y sistémica a la comprensión de cómo se da la acreditación hospitalaria en la organización estudiada. Su estructura consta de tres categorías centrales: 'La forma en que trabajamos', 'La acreditación como instrumento de acción' y 'Amor a la causa'; una categoría de apoyo: 'Desarrollo de profesionales de la salud'; y dos categorías de resultados: 'Excelencia en los resultados' y 'Atención centrada en el paciente'. Estos resultados dialogan con el pensamiento complejo y el estudio de las instituciones.

Palabras clave: Acreditación hospitalaria. Teoría sustantiva. Hospital público. 


\section{INTRODUÇÃO}

Desde sua introdução, na década de 1970, a acreditação se espalhou pelo mundo e se tornou uma parte estabelecida dos sistemas de saúde em mais de 70 países (Greenfield \& Braithwaite, 2008). Dessa forma, é considerada um exemplo de difusão de inovação, em que uma ideia é adotada por alguns, transferida para outros e, em seguida, se torna prática normal (Rogers, 2003).

A acreditação hospitalar, no entanto, não pode ser apenas uma ferramenta de qualidade que se dispõe à adesão de hospitais, pois cada sistema nacional de saúde, com seus arranjos de financiamento, meios de pagamento e instrumentos de coordenação da rede de serviços, apropriará seus procedimentos de acreditação. Assim, são as relações internas da acreditação com os sistemas de saúde que lhe dão sentido, como um amálgama político de qualificação de serviços de saúde que, ao serem moldados para atender a necessidades e limitações de cada país, modifica sua essência e deixa de ser um modelo padrão (Fortes, Mattos \& Baptista, 2011).

É preciso entender que, no formato apresentado no Brasil, a acreditação é um método voluntário, educativo (Monteiro, 2014; Seiffer, 2013; Thimmig, 2007), e, ao mesmo tempo, um sistema de avaliação e certificação da qualidade, com padrões preestabelecidos que aferem a conformidade a um modelo de qualidade na assistência à saúde (Ribeiro, 2006; Seiffer, 2013), capaz de promover mudanças institucionais (Casimiro, 2005) como um mecanismo de incentivo (Jorge, Carvalho \& Medeiros, 2013) que favorece boas práticas e conduz à cultura da qualidade (Seabra, 2007).

Os hospitais brasileiros adotam 3 certificações: a Joint Commission International (JCI), com 39 hospitais acreditados (Consórcio Brasileiro de Acreditação [CBA], 2020); a QMentum International, com 85 hospitais acreditados (Instituto Qualisa de Gestão [IQG], 2020); e a acreditação hospitalar da Organização Nacional de Acreditação (ONA), com 350 hospitais certificados (ONA, 2020).

A ONA é reconhecida formalmente pelo Ministério da Saúde como organização responsável pelo sistema de avaliação da qualidade hospitalar no país, com maior abrangência graças à adaptação de suas normas à realidade brasileira, com observação à legislação nacional (Viana, Sette, Botelho, Rezende \& Poles, 2011), o que torna o instrumento da ONA fundamental para este trabalho. A certificação apresenta 3 níveis conceituais, que conduzem o processo de evolução da qualidade: um vinculado à segurança, outro atrelado à gestão integrada dos processos organizacionais e um terceiro voltado à excelência em gestão (ONA, 2018).

Nesse cenário, outro ponto a ser ressaltado é que, num país com 6.161 hospitais (Datasus, 2020), somente 474 (CBA, ONA \& IQG, 2020) apresentam uma certificação voltada à qualidade da assistência. Ou seja, só $7,8 \%$ dos hospitais brasileiros buscam se vincular, voluntariamente, a um processo de melhoria contínua de sua atividade principal: a assistência prestada ao paciente. Não obstante, as bases de dados indicam um crescimento exponencial a partir de 2014.

Os programas de acreditação na saúde são aceitos por serem considerados um importante motor para melhorar a qualidade e a segurança nas organizações hospitalares (Baker \& Dunn, 2006), mas pouco há publicado sobre o exame transparente dos diferentes aspectos de acreditação e, menos ainda, sobre os resultados ao longo do tempo (Greenfield \& Braithwaite, 2009). Há falta de evidência empírica para examinar o desenvolvimento, a implementação e a evolução da acreditação, que pode oportunamente ser conduzida por pesquisas de base longitudinal e qualitativa (Greenfield, Pawsey, Hinchcliff, Moldovan \& Braithwaite, 2012).

Assim, frente a esse ambiente peculiar chamado hospital, surgiu a motivação de realizar a presente pesquisa, para a qual emergiram 3 lentes de observação: acreditação hospitalar, pensamento complexo e instituições. Essas lentes conduziram à formulação do seguinte problema de pesquisa: como interagem e que fatores influenciam os elementos envolvidos no processo de implantação, manutenção e evolução da acreditação hospitalar?

Com base nessa questão, derivou-se o objetivo do estudo, que é identificar os elementos estruturantes que contribuem para a implantação, a manutenção e a evolução da acreditação hospitalar, bem como as relações entre eles, de acordo com a percepção dos atores sociais envolvidos no processo, e construir conjuntamente uma teoria substantiva capaz de explicar o fenômeno imbricado no processo de acreditação hospitalar. 


\section{PROCEDIMENTOS METODOLÓGICOS}

A opção que melhor pôde respaldar e conduzir este estudo, na busca pela compreensão de um contexto específico, foi o paradigma interpretativo em sua abordagem construtivista.

Ao desenvolver uma teoria substantiva que explica como a acreditação hospitalar ocorre na prática, utilizando a grounded theory, definiu-se a área substantiva como os hospitais acreditados e aportou-se ao estudo um caso prático. Além disso, o método foi usado em todas as suas fases - codificação inicial, codificação focalizada e codificação teórica -, seguindo a vertente construtivista de Charmaz $(2000,2007)$.

A análise foi construída sob entrevistas e observações que ocorreram de forma longitudinal num período de 24 meses, espaço de tempo que compreendeu a manutenção do nível 2 da acreditação hospitalar no HRPT, bem como a evolução ao nível 3 e sua manutenção. ${ }^{1}$ Ademais, a fase anterior, relatada pelos envolvidos ou resgatada de evidências documentais - como relatórios de visitas da ONA -, perfez os 8 anos anteriores. Esse hospital foi construído no nível terciário de atenção à saúde o mais complexo -, numa região que não detinha tal assistência instalada e demandava, até então, o envio da população que necessitava de cuidados de saúde, em nível de média e alta complexidade, para Belém, cujo caminho por terra leva muitas horas, por estradas não asfaltadas - não transitáveis em épocas de chuvas - e/ou em voos de 1,5 hora de duração, para casos extremos.

A análise documental, a observação participante, os relatos nas entrevistas e as conversas de cumbuca contribuíram para a construção, em conjunto com os envolvidos, de um cenário sistêmico, com os fatores que o influenciam, num viés qualitativo. $O$ levantamento de dados foi dividido em 5 rodadas e envolveu 56 participantes. Entre os envolvidos na pesquisa, encontram-se funcionários e pacientes do hospital, representantes da Organização Social de Saúde (OSS)² e consultores ou avaliadores ONA.

O roteiro das entrevistas consistiu numa única pergunta nas primeiras entrevistas e integrou outras surgidas dos contatos estabelecidos, como forma de validar os códigos. As primeiras entrevistas foram realizadas com sujeitos selecionados por ocuparem funções estratégicas.

Houve 2 tipos de entrevistas. Primeiro, entre pesquisadores e entrevistado; na sequência, após a constatação de que os envolvidos trabalhavam algumas questões com brainstorming, o que levou à promoção de reflexões grupais denominadas de "conversa de cumbuca". As conversas de cumbuca tiveram a participação de diferentes atores, entre os quais se destacam diretores, gestores de nível médio e profissionais técnicos. Nesses momentos, os atores sociais eram instigados a refletir sobre pontos que resultaram de entrevistas anteriores e/ou construir associações entre as categorias resultantes.

Houve também a elaboração de um guia de observações, que serviu de direcionador da atenção e da escolha dos episódios a serem observados, assim como da possibilidade de inserção do maior número possível de fatos que poderiam ser vistos e contribuíssem para a compreensão da ação dos envolvidos no contexto. A observação participante (Charmaz, 2009) ocorreu em ocasiões representativas no caminho para a excelência e a manutenção da acreditação hospitalar ONA, bem como em momentos cotidianos, nos quais a ação pôde ser verificada na normalidade.

Este trabalho resultou na construção de uma teoria substantiva, que, após sua elaboração, apresentou interfaces com o conhecimento teórico-empírico prévio, proporcionando pontos de complementaridade e novas lentes acerca do tema "acreditação hospitalar".

\footnotetext{
O hospital foi acreditado em nível 1 em junho de 2010. O processo até o nível 3 demorou 5 anos e meio.

${ }^{2} \mathrm{O}$ HRPT é administrado por uma OSS, ou seja, toda a gestão é realizada por essa organização. Além dos representantes locais que compõem a força de trabalho hospitalar, há suporte de várias áreas por meio de consultorias, assessorias e apoio nos processos. Profissionais que desempenham essas atividades participaram de alguns momentos da pesquisa, sendo entrevistados ou compartilhando ideias em conversas de cumbuca.
} 


\section{APRESENTAÇÃO DO MODELO “COMPROMETIMENTO COM O CUIDADO” E REFLEXÕES À LUZ DAS TEORIAS ADJACENTES: PENSAMENTO COMPLEXO E INSTITUIÇõES}

A teoria substantiva acerca da acreditação hospitalar no HRPT foi construída com base em constatações, reflexões e compartilhamentos de visões que transcorreram das interações entre os diversos envolvidos na pesquisa ao longo de 24 meses. O resultado foi um consenso "nosso" acerca de como a acreditação hospitalar se mostrou possível de implantação, manutenção e evolução num estabelecimento de saúde público, assentado num contexto de recorrentes fragilidades e localizado em meio à região transamazônica brasileira, o que por si só já representa uma barreira ao processo que se desvendou. Os dados da pesquisa conduziram à construção paulatina de um "modelo" que se convencionou chamar de "comprometimento com o cuidado.

No alicerce do modelo constituído, são referenciadas 3 categorias centrais - "a forma como trabalhamos, "acreditação como instrumento de ação" e "amor à causa" -, 1 categoria de suporte - "desenvolvimento dos profissionais de saúde" -, 2 categorias de resultado - "excelência nos resultados" e "cuidado centrado no paciente" -, todas impactadas pelos condicionantes e pelos reflexos do reconhecimento, ambos externos. Essas categorias impactam subcategorias e interagem entre si, formando um quadro geral permeado pelas inter-relações criadas e fortalecidas pelo fazer social instalado, conforme Figura 1.

Figura 1

Modelo "comprometimento com o cuidado"

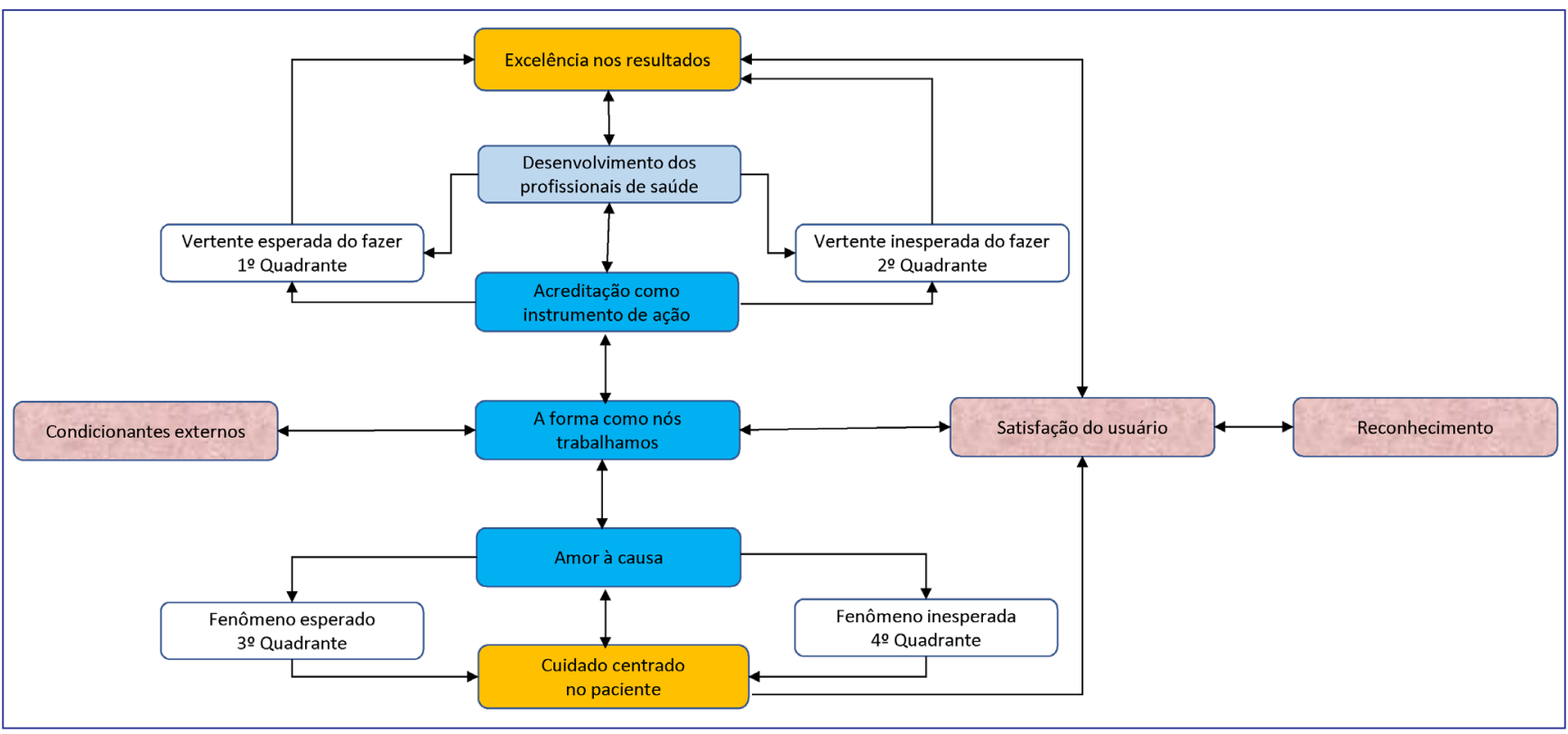

Fonte: Elaborada com base em dados da pesquisa (2018).

A categoria "condicionantes externos" apresenta maior relação explicativa com a vertente econômica dos estudos institucionais (Cavalcante, 2014; North, 1990; Rocha, 2004; Williamson, 1985, 1996), com inferência da vertente sociológica (Daniel, Pereira \& Macadar, 2014; Dimaggio \& Powell, 1983, 1991; Scott, 1995, 2008), ao passo que a categoria central "a forma como trabalhamos" encontra respaldo no pensamento complexo (Agostinho, 2003; Morin, 2002, 2003, 2013; Stacey \& Griffin, 2006) e nos estudos de institucional work (Bruning, Amorim \& Gobri, 2015; Lawrence \& Suddaby, 2006; Lawrence, Suddaby \& Leca, 2011). No pensamento complexo, por entender o ator social e as organizações no ambiente em que interagem; no institucional work, pelo fazer dos indivíduos, por suas ações, atos e formas de entender esse contexto.

Os condicionantes externos - localização, Estado, OSS, sistema de saúde, perfil epidemiológico, Belo Monte, ${ }_{3}$ políticas públicas e sociais, cultura e valores locais - representam a entrada do fazer social do modelo e determinam, modificam e contingenciam

${ }^{3}$ Usina hidrelétrica construída no rio Xingu, que figura entre as maiores do mundo e ocasionou uma mudança drástica no perfil de atendimento. 
o processo instalado e a forma como ele se dá, mediante instituições que o constituem. Assim, a categoria central é impactada a todo momento pelos condicionantes externos, que, nas suas relações com o cenário, determinam, de modo paradigmático, como "a forma como trabalhamos" se posicionará como resposta aos desafios, criando um formato próprio do desenvolver nesse estabelecimento de saúde. "A forma como trabalhamos", como categoria, demonstrou a prática entendida na sua essência e o profundo conhecimento dos gestores da organização, que entendem seus processos, estão cientes de seus gargalos e reconhecem a melhor forma de minimizar o dano, o risco e a insatisfação do paciente acolhido, em conjunto com os demais profissionais do hospital. Logo, esses condicionantes externos servem como uma possibilidade de melhoria.

A acreditação em si é a instituição mais forte no HRPT, talvez aquela culturalmente mais imbricada em todo o processo hospitalar. Instituição é entendida aqui como um hábito mental (Veblen, 1988), por atuar como o tecido social, mantido e modificado por ações e decisões dos sujeitos ao longo do tempo. Mas, ao mesmo tempo, como hábitos de pensamento aceitos como normas orientadoras da conduta (Mitchell, 1910), sem deixar de lado o viés de que a acreditação envolve o processo pelo qual ações sociais e obrigações passam a ter um status de regra no pensamento e na ação social (Meyer \& Rowan, 1983).

A "acreditação como um instrumento de ação" serviu ao HRPT como um manual para estruturar o hospital embasado em qualidade na assistência e em princípios vinculados à segurança, ao foco no paciente, à visão sistêmica, à orientação por métodos e à melhoria contínua. A própria pesquisa empreendida iniciou com o pressuposto de que a acreditação era a ferramenta utilizada para determinar o caminho daquele hospital. Outro entendimento comum foi o de que essa era a perspectiva que dirigia à vertente esperada do fazer, peculiar das organizações que operam motivadas por certificações de qualidade, apesar das nuances do cenário encontrado. Porém, no fazer se encontrou algo que não era esperado e que define a diferença na visão de quem faz e de quem recebe a assistência, ou seja, evidenciou-se que os profissionais que trabalham no HRPT se preocupam em reconhecer seus feitos, em especial aqueles que não são positivos ao processo. Por fazer sentido ao longo da pesquisa, em conjunto, denominou-se esse elemento como a ação inesperada do fazer.

O fazer social, que ilustra o primeiro quadrante do modelo "comprometimento com o cuidado" e representa a "acreditação como instrumento de ação" em sua vertente esperada, é composto pelas categorias "instrumentalização do fazer", "construção interna", "envolvidos no processo", "foco nos processos", "maximização de recursos" e "cultura de qualidade e segurança".

A construção interna se dá por meio de 2 pilares, o primeiro representado pela categoria "envolvidos no processo" e o segundo, pela categoria "instrumentalização do fazer", as quais, em conjunto, formam o que se denominou de triângulo do fazer, um adendo em que se observa a ocorrência dos 3 pilares de influências isomórficas (Scott, 2008): o regulador, o cultural-cognitivo e o normativo. O primeiro se vincula a leis, normas ou sanções que regulam as organizações de saúde e seus profissionais; o segundo apoia as lógicas compartilhadas, que são comuns e se difundiram na forma como o fazer se dá no HRPT; e o terceiro instrumentaliza e diz como as coisas devem ser feitas, integrando a acreditação e apontando o que se espera dos atores sociais envolvidos (Scott, 2008).

O foco nos processos se refere ao modo como a gestão dos processos está instaurada e a quanto a organização é mais ou menos forte do que aqueles que atuam nela, pois a maturidade organizacional chega quando os processos resistem às pessoas, embora se reconheça que os profissionais são peças fundamentais na construção do fazer. Isso deu aporte a 2 movimentos representados pelas subcategorias "maximizar recursos" e "cultura de qualidade e segurança". O primeiro está relacionado à sustentabilidade do hospital e o segundo está ligado a como o serviço prestado apresenta resultados consubstanciados ao usuário do serviço: o paciente. Após a conquista do nível 3, observou-se um cuidado maior com a melhoria e o recurso, que perpassa para além do custo, evoluindo para a consciência de maximizar recursos, como algo que deixa de ser local para ser sistêmico. Desse modo, a ação organizacional pode ocorrer involuntariamente a condicionantes objetivos, racionais ou de eficiência, já que as organizações sobrepujadas às forças de um setor institucional são impelidas a perfilhar práticas socialmente legítimas, aumentando sua expectativa de sobrevivência por meio de usos isomórficos (Dimaggio \& Powell, 1991).

Com relação à cultura de qualidade e segurança, cabe salientar como os envolvidos entenderam que ela se deu. Para tal, a história importa para esse hospital e seus profissionais. Sua trajetória diz como o HRPT é e age, possibilitando que os problemas passados o fortifiquem ao longo dos anos, dando estabilidade à evolução do fazer e dos resultados apresentados. Assim, o caminho se consolidou, de início, com a consciência da segurança do paciente e evoluiu por meio do aprendizado de todos os envolvidos e da constituição de um modelo mental coletivo. A acreditação hospitalar impulsionou concomitantemente esse processo, primeiro com os processos seguros e depois com o evoluir da gestão dos processos e de riscos, seguida por ciclos de melhoria e expressa, finalmente, como qualidade, com a inter-relação dos indicadores, demonstrando 
maturidade organizacional e resultados sistêmicos vinculados à efetividade da ação. Os envolvidos percebem o amadurecimento organizacional, ao mesmo tempo que observam um novo caminho a seguir, o que demonstra a lógica da melhoria contínua instalada - vista de diferentes formas, dependendo do grau hierárquico do envolvido.

O segundo quadrante do modelo comprometimento com o cuidado, também alicerçado pela categoria central "acreditação como instrumento de ação" em sua vertente inesperada, é composto pelas categorias "reconhecimento das fragilidades", "fazer o certo", "corrigir erros", "mitigar riscos", "driblar as limitações" e "estratégia na prática como ação cotidiana".

A vertente inesperada do fazer foi qualificada como a operação para além da parte técnica. Aqui, o contexto é fundamental para o entendimento, visto que o percurso se inicia no âmago da localização do hospital e dos determinantes externos que tornam o HRPT um caso ímpar. Assim, a categoria de saída corresponde ao reconhecimento das fragilidades que são postas ao HRPT e que vão de recursos à cultura. Essas fragilidades são fortalecidas pelo que se denominou círculo motor, que são as saídas geradas do reconhecimento das fragilidades, em que há o propósito de fazer o certo. Para tal, é vital corrigir erros, que será o apoio para mitigar riscos e, de tal modo, driblar as limitações.

Além de concernir ao lado assistencial, que não é o foco neste trabalho, essas fragilidades se vinculam aos custos de transação e ao grau que exigem dos ativos específicos, ou seja, os ativos especializados que não podem ser reempregados sem sacrifício do seu valor produtivo se contratos encerrarem ou forem afetados de forma prematura (Williamson, 1985). No entanto, o que se revelou considerável na pesquisa é que os envolvidos reconhecem que as fragilidades existem, ao mesmo tempo que entendem que elas podem ser transpostas, mesmo com a necessidade de estratégias para ultrapassar os obstáculos.

A próxima categoria desse quadrante, a "estratégia na prática como ação cotidiana", é contrária àquela comum nas organizações, que é pensada, gestada e posta em prática. Ela diz respeito ao fazer cotidiano, como se as fragilidades e os determinantes externos houvessem gerado um mecanismo que possibilita que essa prática seja corriqueira, cotidiana.

Essa realidade alinha com os ambientes instáveis e complexos, como o caso hospitalar, em que a diferenciação entre formulação e implementação de estratégias, na maioria das vezes, é eliminada (Meyer, Pascuci \& Murphy, 2012). Isso ocorre porque, nesses contextos, o formulador passa a ser o próprio implementador, ou, ainda, pelo fato de os implementadores estarem responsáveis pela formulação de suas estratégias.

A categoria de suporte "desenvolvimento dos profissionais de saúde" tem alicerces nas categorias "como estratégia organizacional impulsionada pela acreditação" e "por necessidade local". A significativa rotatividade e o despreparo profissional levaram o HRPT à compreensão de que o aprendizado carecia de tradução em processos seguros e perenes, para além das pessoas, e essa foi a estratégia adotada desde o princípio e que se mantém até hoje, como um processo permanente de educação para a manutenção e a evolução dos resultados. A ação do treinar foi, por necessidade local, consubstanciada pela localização do hospital, pela insuficiência de escolas formadoras na área da saúde e por haver poucos profissionais na região, cuja formação era insuficiente para um hospital planejado para atender média e alta complexidade em patamares elevados de qualidade. Apesar de necessidade local ser um ponto implícito na maioria das organizações, no HRPT ela ocorre de forma exacerbada e trouxe um impacto positivo que apoia o reconhecimento das fragilidades e gira o círculo motor, solidificando o fazer numa estratégia na prática como ação cotidiana. Como forma de compensação, foi instaurada no HRPT outra vertente de sustentação para a educação permanente, traduzida na categoria "como estratégia organizacional impulsionada pela acreditação", agindo como um instrumento de apoio ao processo instituído ao longo da inicialização, da manutenção e da evolução dos níveis da certificação.

Para obter acreditação, é necessário ensinar como isso ocorre, e, de maneira longitudinal, tem-se a evolução dos treinamentos conforme os níveis experimentados. Durante o nível 1, o foco foi em treinamentos de segurança; no nível 2, em treinamentos de interação de processos; no nível 3, em análises críticas e inter-relação de indicadores. Atualmente, os treinamentos se referem ao processo sistêmico de resultados. Sob essas lentes, percebe-se a disseminação do processo da qualidade como um direcionador da estratégia que a impulsiona.

Com isso, observa-se o reforço do pilar normativo de Scott (2008), que aponta o que se espera dos atores sociais envolvidos, suas atitudes e performances em acordo com prescrições de ação e comportamento imputadas pela acreditação hospitalar. Ao mesmo tempo, a mudança traduzida na evolução dos níveis institucionais continua sendo importante, mas o que é relevante nessa categoria é a velocidade com que os indivíduos processam essa mudança. Assim, quanto maior a capacidade dos indivíduos de absorver modelos mentais positivos ao desenvolvimento, maior o potencial para o desenvolvimento (Cavalcante, 2014). 
Tais categorias de desenvolvimento indicam que a categoria "excelência nos resultados" tem respaldo do conhecimento adquirido pelos profissionais de saúde, que, além de mais capacitados, observam a assistência por lentes de qualidade e segurança.

Assim, a categoria de resultado "excelência nos resultados" demonstra a evolução da qualidade da assistência, num processo que se iniciou com a decisão de habilitar a acreditação hospitalar como guia condutora do processo no HRPT. A excelência nos resultados perpassa os números apresentados, que conduzem a uma melhoria da qualidade assistencial e, doravante, tem um fundo de percepção que vai além dos números e necessita de vivência na área onde ocorre para aferir explicação plausível.

No caso analisado, os dados foram lapidados lentamente e, com o tempo, se apresentaram melhores, mais consistentes e condizentes com o nível de excelência. A excelência nos resultados estava obscura, não visível aos olhos, mas estava posta. Faltava cerzir os retalhos do tecido em que fora instaurada; havia um quê da complexidade que não era totalmente clarificado aos olhos dos que conduziam, construíam e movimentavam aquele processo. No entanto, os resultados em 2017 foram distintos, o fazer se iluminou e os profissionais compreenderam que a excelência nos resultados era um processo efetivamente sistêmico, que foi adquirido por meio das experiências vivenciadas/aprendidas e (agora) geridas por cada um e em conjunto, dentro de um processo histórico, que se fez aparente quando alcançaram o entendimento de que o todo é a soma de cada parte integrada.

Assim, quando os resultados proporcionaram evolução de desempenho com tendências favoráveis, os envolvidos no processo perceberam a diferença na forma do fazer, em que a acreditação como instrumento de ação, somada ao desenvolvimento dos profissionais de saúde em suas vertentes esperada e inesperada, pode ser traduzida efetivamente na excelência nos resultados, desde que adicionadas de uma dose de amor.

A categoria central "amor à causa" traz o lado bom do ator social e, com ele, o suporte para os quadrantes inferiores do comprometimento com o cuidado. Os achados deste estudo conduziram a um ponto que não é comum nos exames organizacionais, mas que demonstrou influir, de forma holística, em todos os componentes do comprometimento com o cuidado, sobrepondo a técnica, o dever com a profissão, a responsabilidade organizacional e o cunho social. Instituiu-se, assim, o amor à causa como categoria central que apoia a ação.

Essa categoria é composta, de um lado, pelo quadrante designado pelo conhecimento técnico que encontra o amor, aquele que é considerado um fenômeno esperado do hospital por sua missão, e, de outro, pelo quadrante com apelo emocional e que demonstra a doação pessoal dos envolvidos à assistência hospitalar, denominado de fenômeno inesperado. A soma desses quadrantes dá origem ao que os envolvidos na pesquisa consideram o projeto de suas vidas. É um sentimento que remete à complexidade do pensamento dialógico apresentado por Morin $(2002,2010)$, que une ao mesmo tempo a técnica e o sentimento, o fazer e o sentir, o fenômeno a uma causa, a qual os envolvidos no estudo consideram a de suas vidas.

O terceiro quadrante do comprometimento com o cuidado, vinculado ao amor à causa em seu fenômeno esperado, é circunspeto pelas categorias "garantir a segurança assistencial", "estar junto ao paciente", "proximidade com a comunidade e o sistema de saúde" e "servir de solução à rede de saúde".

A categoria "garantir a segurança assistencial", uma das mais representativas do processo, certamente estaria presente por representar princípios imbricados nos fundamentos de gestão em saúde propostos pela ONA, nomear boas práticas assistenciais e, nos últimos anos, estar atrelada às metas internacionais de segurança que a Organização Mundial da Saúde (OMS) promulga como vitais à assistência segura.

Para garantir a segurança assistencial e estar junto ao paciente, porém, é fundamental exprimir a empatia despertada pela circunstância em que esse se encontra no momento que procura por um serviço de saúde. Estar junto ao paciente abarca a ação para além do que é plausível, em que os profissionais transpassam todos os ínterins para ofertar o cuidado, inovando na forma de fazer, nas soluções, e garantindo solidariedade.

A proximidade com a comunidade e o sistema de saúde torna o hospital uma das principais referências em Altamira, o qual vem desenvolvendo ações sociais, educativas e projetos para além dos muros. Além disso, é um dos maiores ou o maior empregador local, bem como contribui consideravelmente para a economia local. Estar junto com o paciente, aliado à proximidade com a comunidade e o sistema de saúde, é reforçado quando o HRPT desenvolve atividades focadas na comunidade, com o intuito de garantir a continuidade do cuidado dos pacientes que estiveram internados, a prevenção do cidadão e o papel do hospital no contexto de rede de saúde. Assim, o trabalho institucional se dá no esforço intencional para afetar uma instituição ou um conjunto de instituições, em que os institutional workers agem de maneira consciente do que é pretendido (Bruning, Amorim \& Gobri, 2015). 
Como que derivada da proximidade com a comunidade e sistema de saúde, voluntária ou involuntariamente, surge a categoria "servir de solução à rede de saúde", contribuindo com o papel social e assistencial do HRPT, ultrapassando o que Ihe é estipulado como desempenho dentro do sistema de saúde brasileiro: oferecer assistência de média e alta complexidade aos pacientes referenciados.

O quarto quadrante do comprometimento com o cuidado, ligado ao amor à causa em seu fenômeno inesperado, agrupa as categorias "hospital tem significância para as pessoas", "possibilidade de fazer o bem", "envolvimento dos profissionais", "princípio de pertencimento", "resiliência dos grupos assistenciais", "relação afetiva" e "sentimento de realização".

Tem-se, nessa categoria, o somatório do significado atrelado ao local com a atividade desenvolvida no hospital, que é cuidar de pessoas, o que possibilita a visão do lado bom do ser humano. A possibilidade de fazer o bem tem um apelo fraternal, no qual pacientes e profissionais se apoiam na busca de um fazer melhor, de um cuidado mais humano. A própria profissão tem esse viés, porém, na visão dos entrevistados, com o tempo, a automação do cuidar torna o resultado mais operacional e mecânico do que humanizado e centrado no paciente; a empatia desaparece na prescrição do método, o protocolo. Logo, a possibilidade de fazer o bem vem iluminar o caminho inverso. Assim, surge a similaridade com a ideia de processo evolutivo de Veblen, movida pelo instinto denominado parental bent, ou seja, aquele que inclinaria o sujeito a buscar a melhora do bem-estar da família e da sociedade (Cavalcante, 2014; Rutherford, 1984), em que o cuidado se instala e reforça o lado humano dos profissionais.

A assistência prestada é constantemente ajuizada para conferir se o que se faz é o melhor no crivo daqueles envolvidos, para além da visão externa dos órgãos reguladores ou da avaliação da ONA. A acreditação é vista como um instrumento, mas não é o que constrói as categorias voltadas ao amor à causa, que são abastecidas pelo compromisso com o cuidado. A acreditação deu muito ao hospital em técnica e conhecimento, mas o que se estabeleceu é fruto da experiência de um agrupamento local, vivenciado ao longo dos anos, que instituiu outras formas de fazer a assistência, em especial implantando diretrizes vinculadas ao sentir/à união/ao amor.

Percebe-se, portanto, que ações habituais fomentam esse fazer social e estão emaranhadas entre o que é formal e o que é informal, o que é esperado e o que é inesperado, como uma forma de averbar que a realidade tem precedentes sustentados em sentimentos, que podem favorecer seu desenvolver ou não, numa linha sólida entre os opostos. Além disso, pontos como esses favorecem o trabalho, traduzido pelos envolvidos em alegorias como time de trabalho e vestir a camisa, que definem a união desses profissionais. Logo, o desempenho apresentado vai ao acordo da coesão dessas equipes, e o princípio de pertencimento se mostra fundamental.

A possibilidade de fazer o bem, somada ao envolvimento dos profissionais de saúde e ao princípio de pertencimento, fortalece o surgimento de um sentimento de realização, explicitado durante a pesquisa nos momentos em que expressam os diversos pontos de fragilidade para, a seguir, vibrar com as vitórias alcançadas. O sentimento de realização encontra força na possibilidade de crescimento que o hospital proporcionou/proporciona a muitos de seus funcionários. Ademais, o profissional se realiza ao se ver progredindo, o que o motiva à melhoria contínua - própria e da organização -, dá entusiasmo à atuação, eleva o moral da equipe, contribuindo, em última instância, para o cuidado centrado no paciente.

A categoria de resultado "cuidado centrado no paciente" evidencia o lado da humanização do fazer como efeito do processo assistencial. Essa categoria apresentou um forte vínculo com o lado emotivo dos quadrantes relacionados aos fenômenos vinculados ao amor à causa, o que denota alinhamento com o pensamento complexo. Possibilitou, assim, uma resposta ao questionamento que Morin faz no célebre Método 5 - "Será possível transformar a hominização em humanização"? (Morin, 2007)-, pois os resultados encontrados demonstraram o apelo que o local instiga nos envolvidos, oferecendo a esses profissionais a possibilidade de autorrealização por meio do fazer, e, como tal, tornam o que fazem mais humano, oferecem ao outro um cuidado centrado em suas necessidades. Uma possível explicação do que torna essa categoria de resultado tão expressiva é o entendimento dos envolvidos de seu papel naquele contexto. Ao se observarem afastados geograficamente, com poucos recursos de saúde locais e dificuldade de deslocamento e assistência, percebem que o hospital, se colocados em risco de vida, é o que têm de melhor para si e para os seus, o que os leva a manter e melhorar seus processos.

As pessoas se entendem pertencentes ao local onde moram e ao hospital onde atuam, bem como aceitam a certificação de acreditação da ONA como ferramenta que lhes demonstrou como ser melhor, como um guia que lhes possibilitou ir além, fazer melhor pelos outros e por si mesmos, promovendo o cuidado centrado no paciente. Assim, ao mesmo tempo que parece uma solução milagrosa que eleva o desejo de trabalhar para um mundo melhor (Fortin, 2005), enfatizando o sentimento de 
pertencimento do grupo ao hospital e à Altamira, traz a dimensão regulativa do isomorfismo (Scott, 1995, 2008), associada a prescrições e normas impostas pela concepção do desejável, junto com a construção de padrões que demonstram como as coisas devem ser feitas, com o pressuposto de que são "meios legítimos para perseguir fins válidos" (Scott, 1995).

As categorias que encerram o comprometimento com o cuidado são "satisfação do usuário" e "reconhecimento" dos profissionais, do hospital e do processo -, oportunizando a inclusão da visão dos stakeholders acerca da qualidade e da segurança assistencial.

Acerca do usuário principal, que representa o paciente efetivamente atendido no hospital e seus familiares, tem-se a satisfação, aferida internamente pelo Serviço de Atendimento ao Usuário (SAU), que, além de constatar a qualidade instaurada pelo serviço na perspectiva do paciente, atua como mediador quando há problemas ou fragilidades constatados durante o atendimento, ou como apoiador na resolução de necessidades levantadas pelo paciente. Esse contato diminui as fragilidades que causam impacto na assistência, bem como os riscos acoplados a não conformidades encontradas, corroborando com altos índices de satisfação.

O reconhecimento do hospital se dá quando ele passa a ser considerado benchmarking por seus pares que atuam no mercado de saúde brasileiro e percebem nele uma possibilidade para além do que já empreendem em suas organizações hospitalares. Esse tipo de reconhecimento, dito sucesso, é tido como uma influência mimética no campo organizacional, visto que, ante às incertezas a respeito da padronização da assistência por meio da acreditação, despontou essa experiência positiva no ambiente nacional (Daniel et al., 2014).

O reconhecimento do processo se dá quando suas atividades calham de ser referência para outras organizações, ao serem referenciadas em trabalhos científicos como cases de sucesso e/ou quando seus indicadores figuram como comparativos de excelência, servindo de referencial a outras organizações. 0 mesmo vale para o reconhecimento dos profissionais que mantêm, melhoram e inovam as técnicas empreendidas na atividade, acumulando aprendizado e conhecimento acerca da atividade hospitalar, tornando-se distintos no mercado de trabalho - e elevando seu "passe" -, atuando como referenciais dentro das categorias profissionais de atuação. Nessa mesma linha de percepção, Agostinho (2003) argumenta que as relações dos atores residem na reciprocidade entre os indivíduos que, ao buscarem pelo interesse próprio, se apoiam na cooperação. Logo, o próprio reconhecimento fortalece e é fortalecido pelos profissionais por meio dos outros - por exemplo, os pacientes e a comunidade atendida.

\section{CONCLUSÃO}

A primeira etapa do objetivo desta pesquisa foi identificar os elementos estruturantes que contribuem para a implantação, a manutenção e a evolução da acreditação hospitalar. Seu atendimento iniciou com a construção de 9 categorias, agrupadas pelos envolvidos na pesquisa com base na relação que estabeleceram entre os códigos previamente obtidos das coletas de dados. Depois, com o avançar do estudo, desvelou-se que a acreditação hospitalar se estruturava pelo impacto dos determinantes externos, pela forma como os envolvidos realizavam suas atividades, pelo uso da acreditação como instrumento de ação, pelo desenvolvimento dos profissionais da saúde, pela excelência nos resultados, pelo amor à causa e pelo cuidado centrado no paciente.

A segunda parte do objetivo diz respeito a reconhecer as relações entre os elementos estruturantes de acordo com a percepção dos envolvidos no processo de acreditação hospitalar (atores sociais). Assim, os envolvidos no processo apontaram as relações entre as categorias por meio de fios entremeados de complexidade, sendo que, pelos condicionantes externos, em especial da localização e do poder do Estado, é determinada a forma como trabalhamos, a qual é impactada, de um lado, pela técnica da acreditação como instrumento de ação com suas vertentes esperadas e inesperadas do fazer que garantem a excelência nos resultados e, de outro, pelo amor à causa, que em seus fenômenos, esperado e inesperado, propicia o cuidado centrado no paciente, reflete na satisfação do usuário e resulta em reconhecimento do processo, dos profissionais e do hospital. 
Na terceira e última parte do objetivo, propôs-se construir, em conjunto com os envolvidos no processo, uma teoria substantiva capaz de explicar o fenômeno entrelaçado no processo de acreditação hospitalar. Assim, foi construído um modelo sistêmico denominado "comprometimento com o cuidado", que é constituído de um eixo central, por onde se tem as entradas oriundas do cenário externo, a prática do fazer organizacional e o resultado desse processo dentro e fora do contexto hospitalar. Para dar suporte ao modelo, há 3 categorias centrais - "a forma como trabalhamos", "acreditação como instrumento de ação" e "amor à causa" -, 1 categoria de suporte - "desenvolvimento dos profissionais de saúde" - e 2 categorias de resultado "excelência nos resultados" e "cuidado centrado no paciente" -, que são impelidas pelos condicionantes e pelos reflexos do reconhecimento externo. Essas categorias impactam nas subcategorias e entre si, alinhavando o referido esquema, o qual é permeado pelas inter-relações criadas e fortalecidas pelo fazer cotidiano instalado.

Novas categorias de análise foram estabelecidas neste estudo, as quais contribuem para o entendimento da acreditação hospitalar. Tais resultados permitiram olhares para além do mainstream e possibilitaram respostas a pontos que não foram abarcados nos estudos anteriores acerca de organizações hospitalares de alto desempenho. Assim, chegou-se à conclusão de que esses assuntos figuram entre os principais fatores que fizeram com que o HRPT se firmasse como um caso no qual a acreditação encontrou chão para implantação, manutenção, modificação e evolução. Entre tais categorias estão os determinantes externos, em especial o Estado, a cultura e a localização, além da forma como os envolvidos agem, a estratégia como uma prática cotidiana, a proximidade com a rede e, consequentemente, com o sistema de saúde, e o elo criado entre as práticas favorecidas pelo amor à causa e a significância atribuída ao local e à organização.

O conhecimento oriundo da teoria substantiva construída se refere especificamente ao fenômeno estudado na unidade da área substantiva: o HRPT. Embora não generalista, o comprometimento com o cuidado serve para uma leitura mais robusta da complexidade do sistema e possibilita aos técnicos possíveis adaptações. Portanto, parte da teoria ou das categorias específicas podem ser adaptadas para uso pela maioria das organizações hospitalares, acreditadas ou não. Na continuação deste trabalho e no sentido de gerar um alargamento da função proposta pela ONA - e, consequentemente, gerar melhorias ao sistema de saúde brasileiro -, sugerem-se estudos conectados aos elos fortalecidos na cadeia da assistência da rede de saúde, proporcionados pela acreditação. Estudos sobre esses aspectos encontrariam respaldo no pensamento complexo e no institucional work.

Além disso, estudos focando em custos de transação poderiam ser aproximados do mesmo ambiente para reflexões aprofundadas, visto que nesta pesquisa foram parcialmente observados. No entanto, percebeu-se que há espaço para novas contribuições, em especial no que tange à incerteza do ambiente causada pela localização e por seus determinantes. 


\section{REFERÊNCIAS}

Agostinho, M. C. E. (2003). Administração complexa: revendo as bases científicas da administração. RAE-Revista de Administração de Empresas, 2(1), 1-18.

Almasabi, M. H. (2015). The impact of accreditation on quality of care in accredited public hospitals in Saudi Arabia: a mixed methods study (Doctoral Dissertation). Monash University, Melbourne, Australia.

Baker, S., \& Dunn, D. (2006). Accreditation: the hallmark of educational quality. Radiol Technol, 30, 78-123.

Bruning, C., Amorim, A. L., \& Godri, L. (2015). Institutional Work: uma ponte entre estudos críticos e institucionais? Revista Pensamento Contemporâneo em Administração, 9(4), 144-156.

Casimiro, C. F. (2005). A mudança organizacional em um estabelecimento de saúde: um estudo da preparação para acreditação (Dissertação de Mestrado). Universidade do Estado do Rio de Janeiro, Rio de Janeiro, RJ.

Cavalcante, C. M. (2014). A economia institucional e as três dimensões das instituições. Revista de Economia Contemporânea, 18(3), 373-392.

Charmaz, K. (2000). Grounded Theory: objectivist and constructivist methods. (pp. 509-535). In N. K. Denzin, \& Y. S. Lincoln (Eds.), Handbook of qualitative research (2a ed.). Thousand Oaks, CA: Sage.

Charmaz, K. (2007). Constructionism and Grounded Theory. In D. J. F. Gubrium, \& J. A. Holstein (Eds.), Handbook of Constructionist Research (pp. 397-412). New York, NY: Guilford.

Charmaz, K. (2009). A Construção da Teoria Fundamentada: guia prático para análise qualitativa. Porto Alegre, RS: Artmed.

Consórcio Brasileiro de Acreditação. (2020). Unidades acreditadas no Brasil. Recuperado de http://www.cbacred.org.br/unidadesacreditadas-no-brasil.asp

Daniel, V. M., Pereira, G. V., \& Macadar, M. A. (2014). Perspectiva institucional dos sistemas de informação em saúde em dois estados brasileiros. Revista de Administração Contemporânea, 18(5), 650-669.

Datasus. (2020). Cadastro Nacional dos Estabelecimentos de Saúde. Recuperado de http://tabnet.datasus.gov.br/cgi/tabcgi.exe?cnes/ cnv/estabbr.def

Dimaggio, P. J., \& Powell, W. W. (1983). The iron cage revisited: institutional isomorphism and collective rationality in organizational fields. American Sociological Review, 48(2), 147-169.

Dimaggio, P. J., \& Powell, W. W. (1991). Introduction. In W. W. Powell, \& P. J. Dimaggio (Eds.), The new institutionalism in organizational analysis (pp. 1-38). Chicago, IL: The University of Chicago Press.

Fortes, M. T., Mattos, R. A., \& Baptista, T. W. (2011). Acreditação ou Acreditações? Um estudo comparativo entre a acreditação na França, no Reino Unido e na Catalunha. Revista Associação Médica Brasileira, 57(2), 239-246.

Fortin, R. (2005). Compreender a complexidade: introdução ao Método de Edgar Morin. Lisboa, Portugal: Instituto Piaget.

Greenfield, D., \& Braithwaite, J. (2008). Health Sector Accreditation Research: a systematic review. International Journal for Quality in Health Care, 20(3), 172-183.
Greenfield, D., \& Braithwaite, J. (2009). Developing the evidence base for accreditation of healthcare organisations: a call for transparency and innovation. Quality Safety Health Care, 18, 162-163.

Greenfield, D., Pawsey, M., Hinchcliff, R., Moldovan, M., \& Braithwaite, J. (2012). The Standard of Healthcare Accreditation Standards: a review of empirical research underpinning their development and impact. BMC Health Services Research, 12, 329-329.

Instituto Qualisa de Gestão. (2020). Instituições Acreditadas. Recuperado de https://www.iqg.com.br/instituicoes-acreditadas/

Jorge, M. J., Carvalho, F. A., \& Medeiros, R. O. (2013). Esforços de inovação organizacional e qualidade do serviço: um estudo empírico sobre unidades hospitalares. Revista de Administração Pública, 47(2), 327-356.

Lawrence, T. B., \& Suddaby, R. (2006). Institutions and institutional work. In S. Clegg, C. Hardy, T. Lawrence, \& W. R. Nord (Eds.), The Sage handbook of organization studies (2a ed., pp. 215-254). London, UK: Sage.

Lawrence, T. B., Suddaby, R., \& Leca, B. (2011). Institutional work: refocusing institutional studies of organization. Journal of Management Inquiry, 20(1), 52-58.

Meyer, W., Pascuci, L. M., \& Murphy, J. P. (2012). Implementing strategies in complex systems: lessons from Brazilian hospitals. Brazilian Administration Review, 9, 19-37.

Meyer, W., \& Rowan, B. (1983). Intitutionalized organizations: formal structure as myth and ceremony. American Jorunal of Sociology, 2, 340-363.

Mitchell, W. (1910). The rationality of economic activity: II. The Journal of Political Economy, 18(3), 197-216.

Monteiro, M. R. (2014). Capacidades dinâmicas para acreditação: evidências no setor hospitalar (Dissertação de Mestrado). Universidade Federal da Paraíba, João Pessoa, PB.

Morin, E. (2002). As duas globalizações: comunicação e complexidade. In J. Machado-da-Silva, \& J. Clotet (Eds.), As duas globalizações: complexidade e comunicação, uma pedagogia do presente (2a ed., pp. 39-59). Porto Alegre, RS: EIPUC-Sulina.

Morin, E. (2003). Da necessidade de um pensamento complexo. In F. M. Martins, \& M. Machado-da-Silva (Eds.), Para navegar no século XXI: tecnologias do imaginário e cibernética (3a ed., pp. 13-36). Porto Alegre, RS: Editora Sulina.

Morin, E. (2007). O método 5: a humanidade da humanidade. Porto Alegre, RS: Sulina.

Morin, E. (2010). Introdução ao pensamento complexo (4a Ed.). Porto Alegre, RS: Sulina.

Morin, E. (2013). A via para o futuro da humanidade. Rio de Janeiro, RJ: Bertrand Brasil.

North, D. (1990). Institutions, institutional change and economic performance. Cambridge, UK: Cambridge University Press. 
Organização Nacional de Acreditação. (2018). Manual das Organizações Prestadoras de Serviços de Saúde (Coleção Manual Brasileiro de Acreditação). Brasília, DF: ONA.

Organização Nacional de Acreditação. (2020). Certificações Válidas. Recuperado de https://www.ona.org.br/Organizacoes Certificadas $/ 10$

Ribeiro, N. R. C. (2006). A descontinuidade do programa de acreditação hospitalar no Hospital da Restauração: poderia ter sido diferente? (Dissertação de Mestrado). Universidade Federal de Pernambuco, Recife, PE.

Rocha, W. F., Jr. (2004). A nova economia institucional revisitada. Revista de Economia e Administração, 3(4), 301-319.

Rogers, E. (2003). Diffusion of innovations (5a ed.). New York, NY: Free Press.

Rutherford, M. (1984). Thorstein Veblen and the process of institutional change. History of Political Economy, 16(3), 331-348.

Scott, R. W. (1995). Institutions and organizations. Thousand Oaks, CA: SAGE Publications.

Scott, R. W. (2008). Institutions and organizations: ideas and interests (3a. ed.). Los Angeles, CA: Sage.
Seabra, S. A. K. (2007). Acreditação em organizações hospitalares (Dissertação de Mestrado). Pontifícia Universidade Católica do Rio Grande do Sul, Porto Alegre, RS.

Seiffert, L. S. (2013). Modelo de consultoria interna para o preparo de unidades hospitalares para a certificação de acreditação (Dissertação de Mestrado). Universidade Federal do Paraná, Curitiba, PR.

Stacey, R. D., \& Griffin, D. (2006). Complexity and experience of managing in public sector organizations. New York, NY: Routledge.

Thimmig, R. A. (2007). Aplicação da sistemografia para a elaboração da proposta de um método para acreditação de instituição de saúde (Tese de Doutorado). Universidade Estadual de Campinas, São Paulo, SP.

Veblen, T. (1988). A teoria da classe ociosa: um estudo econômico das instituições. São Paulo, SP: Nova Cultural.

Viana, M. F., Sette, R. S., Botelho, D., Rezende, D. C., \& Poles, K. (2011). Processo de acreditação: uma análise de organizações hospitalares. RAHIS Revista de Administração Hospitalar e Inovação em Saúde, 35-45.

Williamson, O. (1985). Transaction cost economics. In O. Williamson (Ed.), The economic institution of capitalism (pp. 15-42). London, UK: The Free Press.

Williamson, O. (1996). The mechanisms of governance. Oxford, UK: Oxford University Press.

Andreia Maria Berto

ORCID: https://orcid.org/0000-0002-8792-7751

Doutora em Administração pela Universidade Federal de Santa Catarina (UFSC); Professor-tutor da Fundação Getulio Vargas (FGV).

E-mail: andreiamberto@hotmail.com

Rolf Hermann Erdmann

ORCID: https://orcid.org/0000-0002-4094-2268

Doutor em Engenharia de Produção pela Universidade Federal de Santa Catarina (UFSC); Professor titular do Departamento de Ciências da Administração da Universidade Federal de Santa Catarina (UFSC). E-mail: rolf.erdmann@ufsc.br

AnVivian Osmari Uhlmann

ORCID: https://orcid.org/0000-0003-2743-5293

Doutora em Administração pela Universidade Federal de Santa Catarina (UFSC). E-mail: vouhlmann@gmail.com 